\title{
Fermi surfaces and single-particle spectral functions of low-dimensional inorganic non-cuprate compounds: the molybdenum bronzes
}

\author{
G-H Gweon $\dagger$, J W Allen $\dagger$, R Claessen $\ddagger$ J A Clack $\dagger$, D M Poirier§, \\ P J Benning§, C G Olson§, W P Ellis $\|$, Y-X Zhang $\dagger$, L F Schneemeyer $\Phi$, \\ $\mathrm{J}^{\text {Marcus }}{ }^{+}$and C Schlenker ${ }^{+}$ \\ $\dagger$ Randall Laboratory, University of Michigan, Ann Arbor, MI 48109-1120, USA \\ $\ddagger$ Universität des Saarlandes, Fachrichtung 10.2-Experimentalphysik, D-66041, Saarbrücken, \\ Germany \\ $\S$ Ames Laboratory, Iowa State University, Ames, IA 50011, USA \\ || Los Alamos National Laboratory, Los Alamos, NM 89545, USA \\ I Bell Laboratories, Lucent Technologies, Murray Hill, NJ 07974, USA \\ + Laboratoire d'Etudes des Propriétés Electroniques des Solides, CNRS, BP166, 38042 Grenoble \\ Cédex 9, France
}

Received 15 July 1996, in final form 3 September 1996

\begin{abstract}
The Fermi surfaces and single-particle spectral functions of several low-dimensional materials have been measured as part of an effort to assess the occurrence of non-Fermi-liquid behaviour in non-cuprate materials.
\end{abstract}

\section{Introduction}

The paradigm lattice model of non-Fermi-liquid (NFL) behaviour is the one-dimensional (1D) Luttinger liquid (LL) [1], and the Tomonaga-Luttinger (TL) model is an exactly solvable model with LL behaviour [2]. The spectral weight $\rho(\boldsymbol{k}, \omega)$ of the $\boldsymbol{k}$-resolved single-particle Green's function for the LL is very different from that for the Fermi liquid (FL). Figure 1(a) shows $\rho(\boldsymbol{k}, \omega)$ calculated for the FL self-energy, having linear and quadratic $\omega$-dependencies of its real and imaginary parts, respectively, and figure 1(b) shows $\rho(\boldsymbol{k}, \omega)$ for the TL model [3]. The former has a single quasi-particle peak, while the latter shows two peaks, reflecting the fact that removal of an electron results entirely in the generation of holon-spinon pairs. In addition the TL lineshape shows edge singularities at the holon and spinon energies, with power-law tails characterized by exponents usually called $K_{\rho}$ and $K_{\sigma}$, respectively, and for $\boldsymbol{k} \neq \boldsymbol{k}_{F}, \rho(\boldsymbol{k}, \omega)$ is zero for $\omega$ between $E_{F}$ and the lowest-energy singularity. With attention paid to important technical difficulties, which are minimized in quasi-low-dimensional materials [4], $\rho(\boldsymbol{k}, \omega)$ can be measured via angle-resolved photoemission spectroscopy (ARPES). Thus ARPES spectral lineshapes are in principle a very direct experimental probe for NFL behaviour. Indeed, the slowly falling high-binding-energy tails and other aspects of the ARPES spectra of the quasi-2D superconducting cuprates have been proposed to signal a deviation from FL behaviour, from the viewpoints of the possibility of a 2D LL [5] and also of a marginal Fermi liquid [6].

Motivated by these ideas, we have been engaged in a programme to find clear ARPES examples of Fermi-liquid behaviour and of Luttinger-liquid behaviour in quasi-1D and 


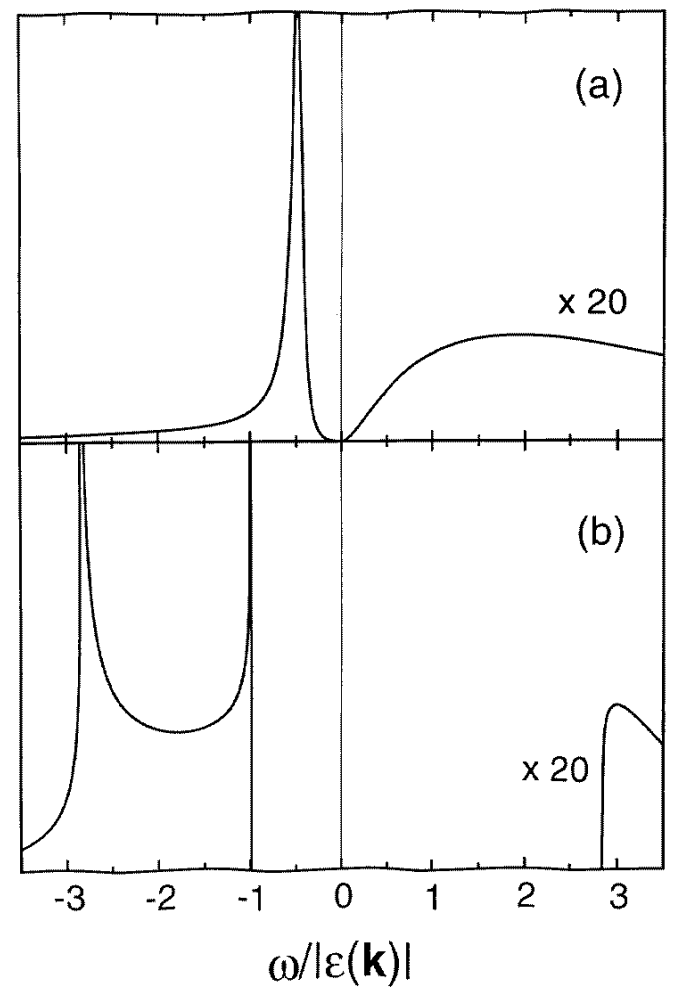

Figure 1. The one-hole spectral function for (a) a FL theory with electron self-energy $\Sigma(\boldsymbol{k}, \omega)=a \omega+\mathrm{i} b \omega^{2}$ and (b) a TL model (adapted from reference [3]) with spin-independent repulsive electron-electron interactions. The energy is defined relative to the Fermi energy and scaled with the non-interacting band energy $\epsilon(\boldsymbol{k})(<0)$. The intensity for the positive $\omega$ is multiplied by 20. The parameters used for this example are (a) $Z=(1-a)^{-1}=0.5$, $Z b \epsilon(\boldsymbol{k})=-0.3$ and (b) $\alpha=0.3, v_{F} /\left(r_{c} \epsilon(\boldsymbol{k})\right)=-0.5$. Here, $Z$ is the mass renormalization factor and $\alpha$ is the so-called 'anomalous dimension'. See reference [3] for the definition of $r_{c}$. Notice the qualitative differences in the number of peaks, peak positions and weight distributions.

quasi-2D materials. With the first of these goals in mind we have already measured and analysed ARPES spectra for quasi-2D 1T-TiTe 2 , as summarized briefly in a later section. In pursuing the second goal, we have been intrigued and encouraged by the result obtained from angle-integrated photoemission spectroscopy (PES) for several quasi-1D materials that the PES spectrum does not show a Fermi edge [7, 8].

One explanation for this vanishing weight at the Fermi energy $E_{F}$ is provided by the ideas of the LL, e.g. the $k$-integrated TL spectrum $\rho(\omega)$. Owing to the low-energy edge singularities in $\rho(\boldsymbol{k}, \omega)$, the onset of $\rho(\omega)$ is not a Fermi edge, but is instead a power law [3] with an exponent usually called $\alpha$. A well known and related property having the same origin is that the occupation number $n_{k}$ shows at $\boldsymbol{k}_{F}$ only a slope singularity, also characterized by $\alpha$, rather than a discontinuity as with a FL. For one organic quasi1D material, the Bechgaard salt (TMTSF) ${ }_{2} \mathrm{PF}_{6}$, there is independent evidence [9] of LL behaviour, and similar $\alpha$-values have been inferred from NMR [9] and PES [8] studies. However, the large $\alpha$-values [10] and the energy scale of the PES onset [11] have been cited as causes for scepticism. 
Inorganic quasi-1D compounds, such as $\left(\mathrm{TaSe}_{4}\right)_{2} \mathrm{I}$ and $\mathrm{K}_{0.3} \mathrm{MoO}_{3}$, also show [7] vanishing $\mathrm{PES}$ weight at $E_{F}$. For these materials, strong electron-phonon interactions are signalled by the presence of charge-density-wave (CDW) transitions which gap the FS below some temperature $T_{p}$. This provides another explanation of the vanishing weightthat the strong CDW fluctuations [12] which can occur for a quasi-1D system, even well above $T_{p}$, act to suppress the weight at $E_{F}$. Indeed a central question for these materials is that of whether or not all of the phenomena observed for them can be accounted for in a CDW scenario [13]. However, recent detailed theory [14] of this scenario casts doubt on its applicability to $\mathrm{K}_{0.3} \mathrm{MoO}_{3}$. Finally, it has been suggested [15] that, at least for some of these materials, surface degradation may be the explanation of the vanishing weight at $E_{F}$.

In the face of these uncertainties there is strong motivation to measure the ARPES spectra of these quasi-1D materials, in order to further characterize the sample surfaces, to verify that a FS can actually be observed, and to search for spectral features signalling LL behaviour. ARPES studies of quasi-2D CDW materials are also important for comparison to the quasi-1D results. In later sections of this paper we summarize our results to date for one of the most important groups of inorganic low-dimensional materials, the molybdenum bronzes. We show sets of ARPES energy distribution curves (EDCs) and also, for two materials, direct Fermi surface images. Not presented here are our results [16] for the inorganic compound $\left(\mathrm{TaSe}_{4}\right)_{2} \mathrm{I}$, which differ in significant ways from the results [17] of a previous study.

\section{Experimental details}

Single-crystalline samples of the molybdenum bronzes studied in this paper were grown by the electrolytic reduction technique $[18,19,20]$. The purple bronzes $\left(\mathrm{AMo}_{6} \mathrm{O}_{17}\right.$, $\left.\mathrm{A}=\mathrm{K}, \mathrm{Na}, \mathrm{Li}_{0.9} \mathrm{Mo}_{6} \mathrm{O}_{17}\right)$ have dimensions of roughly $1 \mathrm{~mm} \times 1 \mathrm{~mm}$, and the blue bronze $\left(\mathrm{K}_{0.3} \mathrm{MoO}_{3}\right)$ is several $\mathrm{mm}$ square. The ARPES experiments were performed at two synchrotron facilities - the Synchrotron Radiation Center (SRC) at the University of Wisconsin and the Stanford Synchrotron Radiation Laboratory (SSRL). Data reported here for $\mathrm{TiTe}_{2}, \mathrm{NaMo}_{6} \mathrm{O}_{17}, \mathrm{KMo}_{6} \mathrm{O}_{17}$ and $\mathrm{K}_{0.3} \mathrm{MoO}_{3}$ were measured at the SRC and those for $\mathrm{Li}_{0.9} \mathrm{Mo}_{6} \mathrm{O}_{17}$ at the SSRL. At the SRC we used the Ames-Montana beamline, equipped with a Seya monochromator. Photons in the approximate energy range $17 \mathrm{eV}-25 \mathrm{eV}$ were used. The photoemitted electrons were energy analysed with a $50 \mathrm{~mm}$ radius hemispherical analyser. At the SSRL, data were taken at the undulator beamline 5. Photons with the energy $20.2 \mathrm{eV}$ were selected. A similar electron energy analyser, modified for multichannel detection, was used. The angle-integrated PES for $\mathrm{NaMo}_{6} \mathrm{O}_{17}$ and $\mathrm{K}_{0.3} \mathrm{MoO}_{3}$ was done at the home laboratory at the University of Michigan with a Vacuum Generator (VG) ESCALAB MK II spectrometer. The 21.2 eV He I line of a He discharge lamp was used as the source for the light.

In all of the experiments, clean surfaces of the single-crystalline samples were obtained by in situ cleaving. The chamber base pressure was $\sim 4 \times 10^{-11}$ at the home laboratory and the SRC and $\sim 2 \times 10^{-10}$ at SSRL. The sample orientations for the ARPES were determined from Laue diffraction photographs. The Fermi energy and the overall energy resolution were determined from the Fermi edge spectra on clean Pt or Ag surfaces prepared by Ar sputtering or on evaporated Au. For each facility, the best combined energy resolution from the photon source and the electron energy analyser was $\sim 35 \mathrm{meV}$ FWHM. When this was unnecessary, resolutions of $\sim 100 \mathrm{meV}$ or $\sim 150 \mathrm{meV}$ FWHM were used in order to obtain higher count rates. The angular resolutions for both ARPES analysers were $2^{\circ}$ full acceptance. The temperature was kept at $20 \mathrm{~K}$ for $\mathrm{TiTe}_{2}$ and at $32 \mathrm{~K}$ for $\mathrm{Li}_{0.9} \mathrm{Mo}_{6} \mathrm{O}_{17}$ in 
order to avoid thermal broadening of the spectra. For $\mathrm{NaMo}_{6} \mathrm{O}_{17}, \mathrm{KMo}_{6} \mathrm{O}_{17}$ and $\mathrm{K}_{0.3} \mathrm{MoO}_{3}$, the measurements were done in the non-CDW phase, by keeping the temperature sufficiently higher than the transition temperature.

For the Fermi surface maps, the ARPES intensity at $E_{F}$ was recorded while the analyser orientation was changed in steps of $1^{\circ}$. Our method is slightly different from the usual one which involves rotating the sample with a fixed analyser. If the sample is fixed, the image is not modulated by the dependency of the photoemission intensity on the orientation of the photon beam. In the sudden approximation of photoemission theory [21], the intensity is given by the product of the photoemission matrix element and the spectral function. Thus we interpret the local maxima of the $E_{F}$ intensity image as showing lines of the FS, but attach no particular significance to the intensity variation across the pieces of FS. This variation arises from the $\boldsymbol{k}$-dependence of the matrix element and the details will be different for different incident angles and energies of the photons.

\section{The quasi-2D Fermi-liquid reference material $\mathrm{TiTe}_{2}$}

$\mathrm{TiTe}_{2}$ is a layered compound which is a semi-metal due to a small energetic overlap of a set of nominally Te p bands and one orbitally non-degenerate $\mathrm{Ti} 3 \mathrm{~d}$ band. Its transport properties give no indication of any behaviour lying outside of the FL framework [22]. In previous work with our collaborators we have measured [23] at low temperature and with high resolution the ARPES spectra of the Ti $3 \mathrm{~d}$ band as it crosses $E_{F}$ along a direction in $\boldsymbol{k}$-space where there are no other bands to produce interfering or overlapping spectral structures. We have confidence in our identification and understanding of this special band because we have verified by ARPES all of the $E_{F}$-crossings predicted by band theory throughout the Brillouin zone [24], and have measured [25] a direct FS image in good agreement with that predicted by density functional calculations. The Ti 3d EDCs are shown in figure 2, all normalized to have the same height, along with theoretical fits described in the next paragraph. The spectra are essentially free of inelastic backgrounds. From top to bottom, one sees the change in the spectral shape as the band disperses from its bottom to cross $E_{F}$. The $\boldsymbol{k}_{F}$-spectrum is identified as being the one with the peak nearest to $E_{F}$ and having the smallest linewidth. The normalized spectra do not show the large decrease in intensity which occurs as the peak crosses $E_{F}$. The bottom two spectra show very small amounts of spectral weight remaining for $\boldsymbol{k}$ outside the Fermi surface, reflecting the presence of hole-particle pairs in the interacting ground state. A physical interpretation of the 'anti-dispersion' of this weight is currently lacking, but the anti-dispersion is well reproduced by both the theories described next.

We have analysed these spectra in detail, first [23] using the simplest FL ansatz in which the self-energy has a $\boldsymbol{k}$-independent FL Taylor's series expansion up to second order in $\omega$. In this ansatz, the truncation of the self-energy renders it non-causal, and a subsequent [26] analysis used a two-parameter phenomenology which keeps the low-energy FL form, but restores the causality of the self-energy in the simplest possible way. This causal ansatz has the interesting property that, for different ranges of values of one of its parameters, it can produce either a single spectral peak, or a structured lineshape. Fits to the data using this ansatz are shown in figure 2. In these fits, the ansatz parameters were allowed to be $\boldsymbol{k}$-dependent so that the interesting shape change from a single peak near $\boldsymbol{k}_{F}$ to a structured lineshape at the bottom of the band could be described. One sees by this example that even in a simple situation, the lineshape need not be simply a single peak which sharpens as it disperses toward $E_{F}$ and then entirely disappears upon crossing $E_{F}$. 


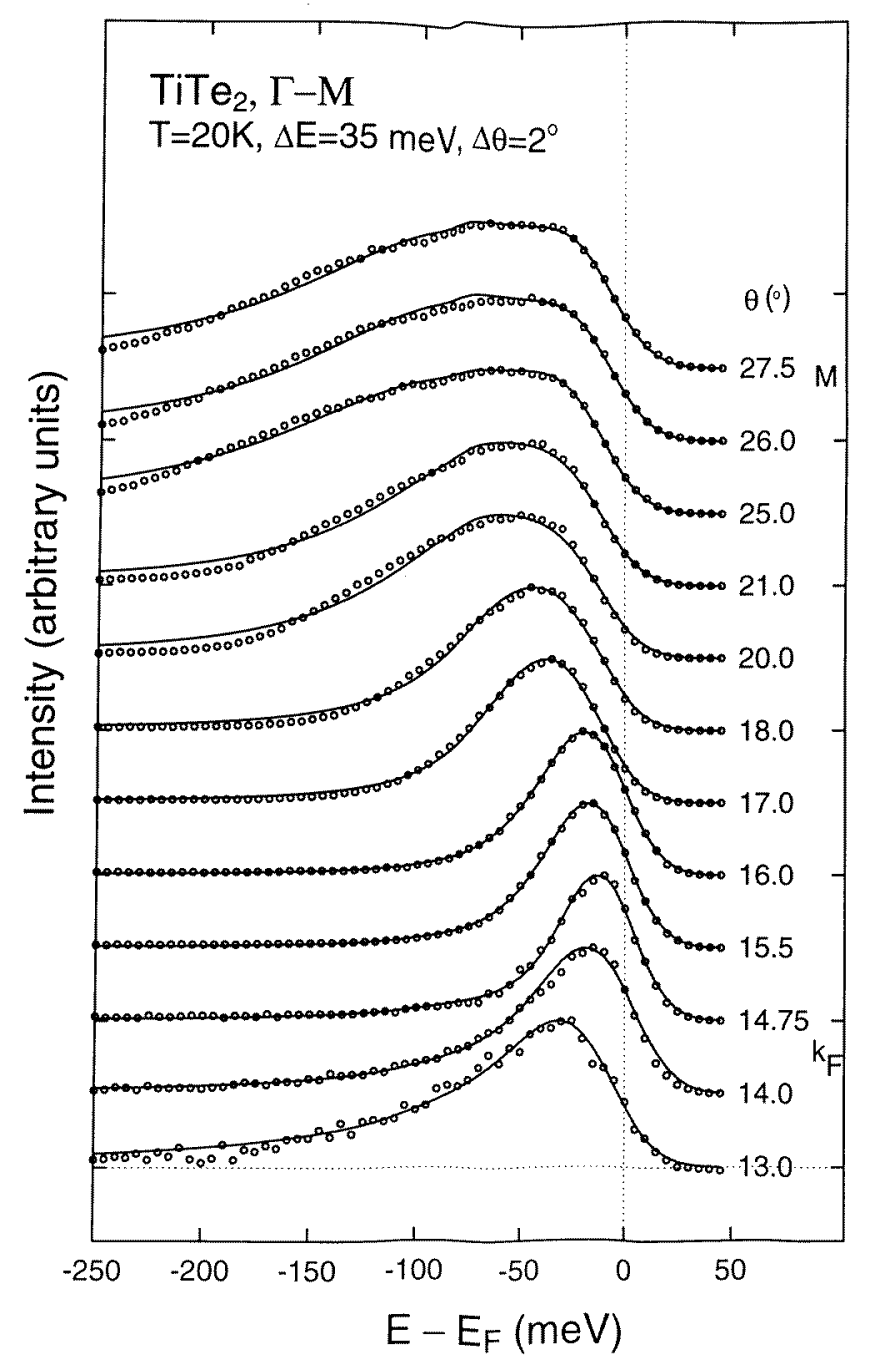

Figure 2. Ti $3 \mathrm{~d}$ ARPES for $\mathrm{TiTe}_{2}$ and a fit with a causal FL self-energy. The spectra are normalized to the same maximum height. See the text and reference [26] for details of the model and the fit.

\section{Molybdenum bronzes}

The molybdenum bronzes are an important family of quasi-low-dimensional materials with the general formula $\mathrm{A}_{x} \mathrm{Mo}_{y} \mathrm{O}_{z}$, where $\mathrm{A}$ is an alkali metal or thallium. As recently reviewed [27], many of these materials display CDW formation where the wavevector $\boldsymbol{q}$ is determined by the FS topology and so can be either commensurate or incommensurate with the underlying lattice. CDW theory shows that the essential requirement is for substantial portions of the Fermi surface to be separated by the same wavevector $\boldsymbol{q}=2 \boldsymbol{k}_{F}$, a circumstance known as 'nesting'. Depending on whether or not the entire FS is gapped when the CDW forms, a metal-semiconductor or metal-metal transition occurs at $T_{p}$. The molybdenum bronzes provide examples of these behaviours. 


\subsection{Quasi-2D potassium purple bronzes}

The so-called potassium purple bronzes, $\mathrm{KMo}_{6} \mathrm{O}_{17}$ and $\mathrm{NaMo}_{6} \mathrm{O}_{17}$, are quasi-2D materials with metal-metal transitions and commensurate CDW formation at $T_{p} \mathrm{~s}$ of $120 \mathrm{~K}$ and $80 \mathrm{~K}$, respectively. Crystallographic studies show that $\mathrm{KMo}_{6} \mathrm{O}_{17}$ is trigonal [18] with space group $P \overline{3}$ and that $\mathrm{NaMo}_{6} \mathrm{O}_{17}$ has only a very slight monoclinic distortion [28] from this symmetry. Their electronic structures are expected to be essentially identical [29], and it is reported [30] that they have the same CDW wavevector.

These materials are particularly interesting in the context of searching for NFL behaviour because [30] their quasi-2D electronic structure can usefully be regarded as being generated from quasi-1D chains forming a pattern of equilateral triangles in the crystal structure. The electronic structure is dominantly determined by dispersion along the chains with only weak hybridization between them. In turn, the FS can be visualized as three pairs of parallel lines, the pairs being oriented at $120^{\circ}$ to one another. The connectivity and final details of the actual FS are determined by small hybridization gaps which open at the crossing points of the lines. By considering the FS in which hybridization is neglected, it is easy to identify a single nesting vector which perfectly connects the two lines of any two of the three pairs. This concept, which has obvious significance for the CDW properties, has been called 'hidden nesting' by Whangbo et al [30], its originators. The $\boldsymbol{q}$ so identified is the CDW wavevector actually observed [30, 31]. Of significance for LL thinking, theoretical studies [32] of the effects of higher dimensionality often consider weakly coupled 1D chains, although usually parallel chains rather than the more complex arrangement considered here.

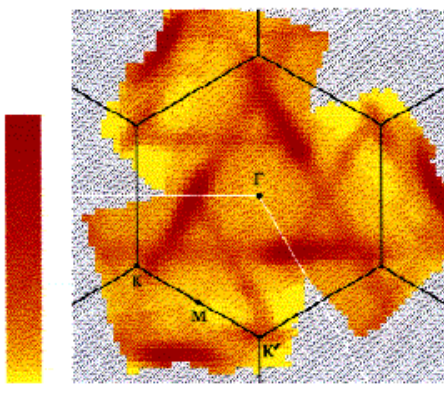

(a)

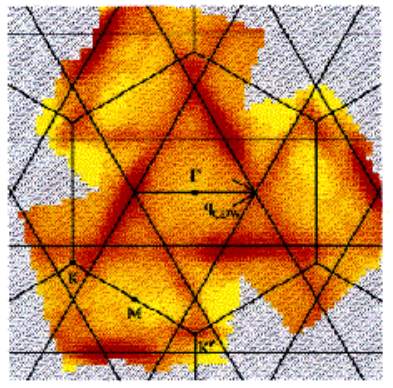

(b)

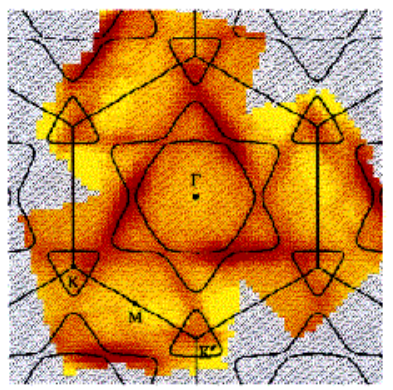

(c)

Figure 3. (a) The Fermi energy intensity map for $\mathrm{NaMo}_{6} \mathrm{O}_{17}$ at $300 \mathrm{~K}$ with photons of energy $22 \mathrm{eV}$, showing Fermi surfaces composed of six nearly straight lines. The overall instrumental energy resolution was $150 \mathrm{meV}$ FWHM. The measured data, whose boundary is shown with thin white lines, constitute one third of the image. The rest was generated by threefold rotations. One cell in the map is approximately one measured data point. (b) A comparison of our data with three quasi-1D FSs for $\mathrm{KMo}_{6} \mathrm{O}_{17}$ (from reference [30]). One of the three equivalent observed CDW wavevectors, from references [30,31], is shown. The optimal nesting condition, nesting of any two pairs of FSs, explains the observed CDW wavevector ('hidden nesting' [30]). (c) A comparison with a $2 \mathrm{D}$ tight-binding calculation including inter-chain hybridization for $\mathrm{KMo}_{6} \mathrm{O}_{17}$ (from reference [30]).

Figure 3(a) shows the direct FS image for $\mathrm{NaMo}_{6} \mathrm{O}_{17}$. A detailed discussion of the procedures for obtaining the image, and a comparison to previous work [33] which obtained a very different result, is given elsewhere [34]. Here we summarize by saying that the one third of the image enclosed by the two white lines represents the actual data set of 783 


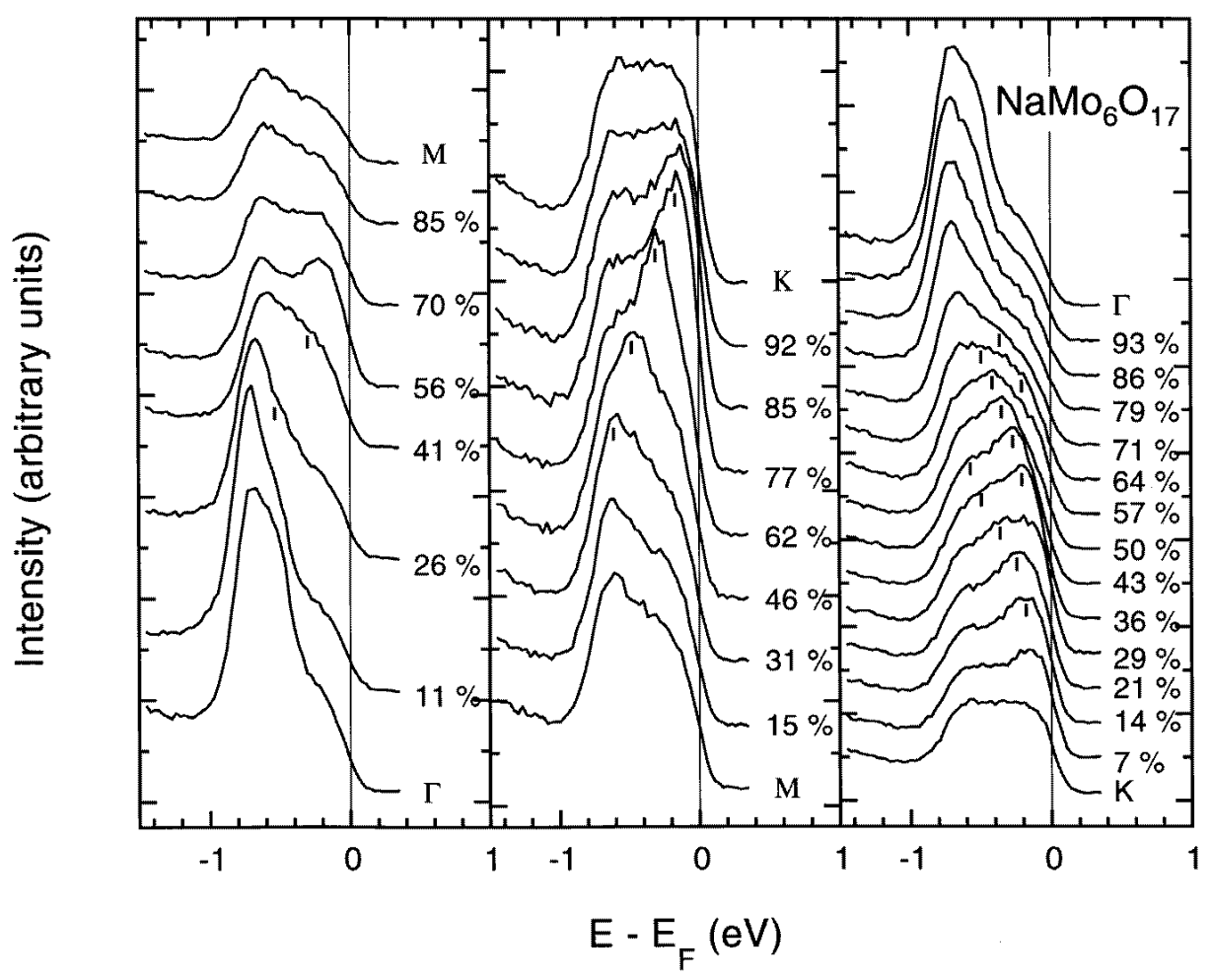

Figure 4. ARPES EDCs obtained for the same surface as in figure 3. The percentage denotes the relative distance along the line in each segment. Tick marks are guides to the eye for dispersing features which can be identified with strong input from the map of figure 3 .

points, and that the complete image was obtained by threefold rotations valid for $P \overline{3}$. The dominant feature of the resulting pattern is three pairs of lines defining a central hexagon. Overplotted in the figure is the appropriate quasi-2D hexagonal Brillouin zone, for which the $\Gamma$-to-M distance is $0.66 \AA^{-1}$. One line in each pair is stronger in intensity and clearly persistent across the entire zone, while the other is weaker and visible only in about two thirds of the central zone. We attribute the details of this intensity variation to the ARPES matrix element. From the hexagonal symmetry of the central image one can be confident that both lines of the FS persist across the entire zone. Indeed, the missing segments would be filled in if we completed the image with the improper sixfold rotations $\mathrm{S}_{6}$, valid for $P \overline{3}$.

Figures 3(b) and 3(c) compare the image with the theoretical FS obtained in tightbinding calculations [30] which neglect and include inter-chain hybridization, respectively. The general level of agreement is nearly quantitative. There are three underlying quasi-1D bands, each half-filled (i.e. occupying half of the first Brillouin zone), with small inter-chain hybridization gaps. The gap along $\Gamma \mathrm{K}$ is resolved in the image, as shown by the slight local minimum at the point where two lines cross near $\mathrm{K}$. The pattern in which a streak actually crosses $\mathrm{K}^{\prime}$ disagrees with the theoretical FS, is inconsistent with the hexagonal symmetry of the central part, and does not connect naturally to the rest of the pattern. Therefore we doubt whether this feature is an intrinsic property of the single crystal and attribute it to a displacement of the bright image nearby, due to picking up some intensity from a part of the surface which may have a slightly different orientation. In this outer part of the image 
where the electron collection angle is large, one is sampling the largest surface area and so is most vulnerable to this problem.

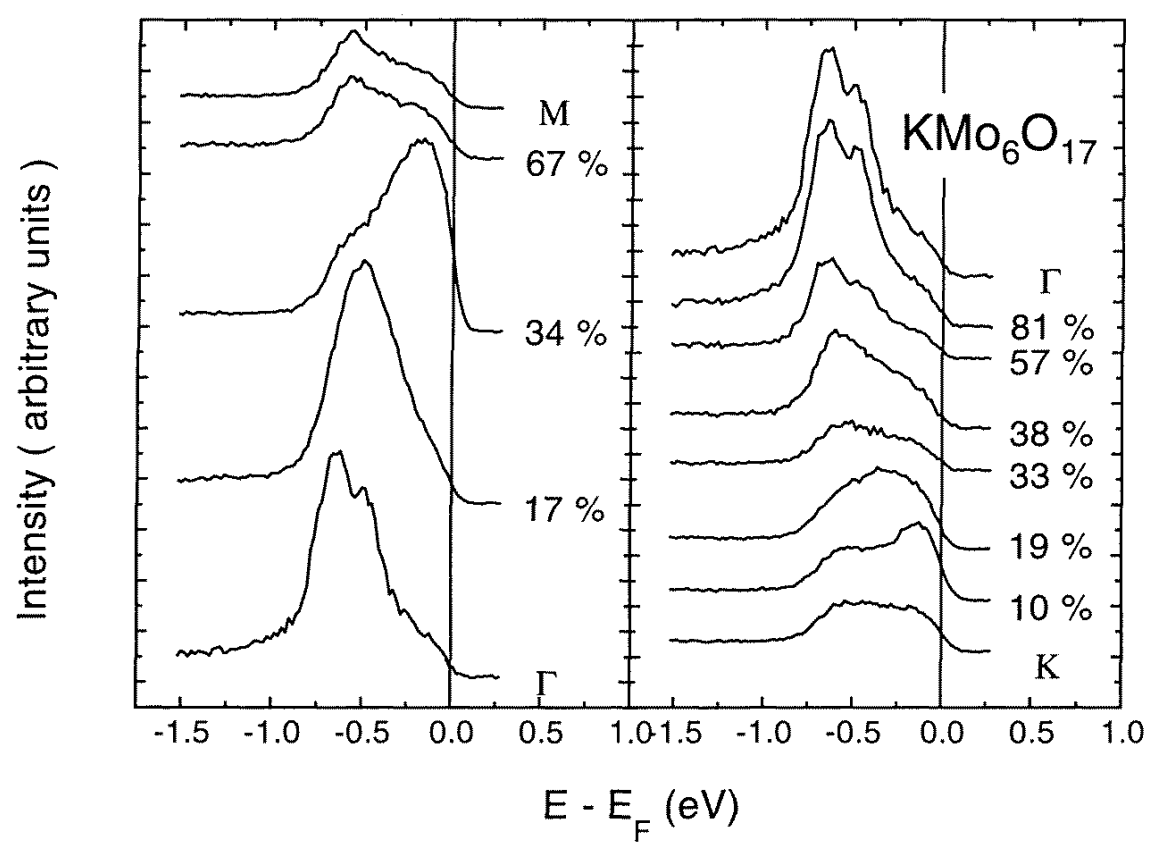

Figure 5. ARPES EDCs for $\mathrm{KMo}_{6} \mathrm{O}_{17}$ at $220 \mathrm{~K}$ with a photon energy of $22 \mathrm{eV}$. The overall instrumental energy resolution was $100 \mathrm{meV}$ FWHM. The spectra show a general similarity with those of figure 4 , although $\boldsymbol{k}$-space is sampled more coarsely.

Figure 4 shows the ARPES EDCs for the same surface and conditions as for the FS image, taken along three lines of high symmetry, the end points of which are marked in figure 3. The FS image shows two unresolved $E_{F}$-crossings at $\sim 55 \%$ of $\Gamma \mathrm{M}$, one crossing at $\sim 86 \%$ of $\mathrm{MK}$, and three crossings at $\sim 12 \%, \sim 29 \%$, and $\sim 60 \%$ of $\mathrm{K} \Gamma$. With this information one can then more easily discern the peak dispersions that lead to these crossings, as shown by the tick marks in figure 4 . But it is nearly impossible to map the entire FS from EDCs with such complex lineshapes and broad overlapping features, and indeed a previous attempt [33] to do so for this material entirely missed one set of quasi1D FS pieces. Figure 5 shows a minimal set of EDCs for the $\mathrm{K} \Gamma$ and $\Gamma \mathrm{M}$ directions of $\mathrm{KMo}_{6} \mathrm{O}_{17}$. Although the number of angles measured is insufficient for observing all of the crossings of $E_{F}$ visible in the spectra for $\mathrm{NaMo}_{6} \mathrm{O}_{17}$, nonetheless it is clear that the spectra of the two materials are generally very similar, as expected.

We can make two further observations from comparing to the tight-binding band calculations [29,30]. First, the total occupied bandwidth calculated, $0.3 \mathrm{eV}$, is about a factor of two smaller than that observed, $0.6 \mathrm{eV}$. We will return to this point in discussing the blue bronze in the next section. Second, we note that, according to the band calculations, there is only a single band along MK. Nevertheless, the lineshapes along MK are complex, always extend across the entire bandwidth, and even show an interesting two-feature structure after the band has crossed $E_{F}$. Further study may be necessary to determine whether the lineshapes actually represent the hole spectral function, but if so, they are a clear sign 
of electron-electron interactions. In fact, there is more than a little resemblance to the complex lineshapes observed for the single $\mathrm{Ti} 3 \mathrm{~d}$ band crossing in $\mathrm{TiTe}_{2}$ (see figure 2) and successfully analysed for that material with the two-pole causal self-energy.

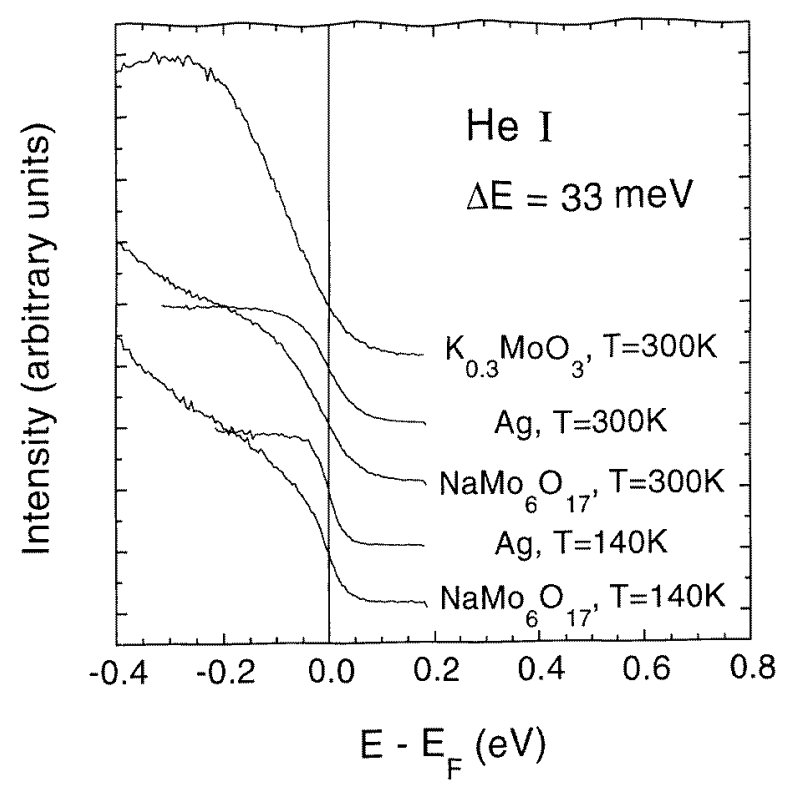

Figure 6. Angle-integrated PES spectra of $\mathrm{K}_{0.3} \mathrm{MoO}_{3}$ and $\mathrm{NaMo}_{6} \mathrm{O}_{17}$ taken at $300 \mathrm{~K}$ and $140 \mathrm{~K}$. The He I line $(21.2 \mathrm{eV})$ was used for the photon source. The overall instrumental energy resolution was $33 \mathrm{meV}$ FWHM. Spectra for Ag are shown for comparison. Note the absence of a Fermi edge only for $\mathrm{K}_{0.3} \mathrm{MoO}_{3}$.

Finally, it is important to examine the angle-integrated PES spectrum near $E_{F}$. This is shown for $\mathrm{NaMo}_{6} \mathrm{O}_{17}$ at $300 \mathrm{~K}$ and $140 \mathrm{~K}$ in figure 6, along with spectra for $\mathrm{Ag}$ at the same two temperatures and a $300 \mathrm{~K}$ spectrum for the quasi-1D blue bronze $\mathrm{K}_{0.3} \mathrm{MoO}_{3}$. It is apparent that the onset for the purple bronze shows a Fermi edge, like the simple FL metal $\mathrm{Ag}$, in sharp contrast to the blue bronze, which lacks a Fermi edge. Consistent with this finding, examination of the purple bronze EDCs shows that for the spectra of the crossings of $E_{F}$, there is substantial weight at $E_{F}$. In this respect the quasi-2D purple bronzes are like the quasi-2D cuprates, even though the two differ in that a considerable body of spectral and transport evidence has been assembled that the latter are NFL materials.

\subsection{Quasi-1D blue bronze}

The 'blue' bronze $\mathrm{K}_{0.3} \mathrm{MoO}_{3}$ is a quasi-1D material with a metal-semiconductor transition and incommensurate CDW formation at $T_{p}=180 \mathrm{~K}$. As reported previously [7] and as confirmed in figure 6 for our samples, its PES spectrum has a smooth onset at $E_{F}$, without a Fermi edge. Tight-binding calculations $[35,36]$ lead to the expectation of two pairs of essentially degenerate bands crossing $E_{F}$ along the quasi-1D direction. The separation of the two pairs is due to weak intra-chain coupling, and the degeneracy within a pair reflects very weak hybridization between chains in the same unit cell. The result is a FS consisting of four lines.

Given the lack of a Fermi edge in the PES spectrum, one may wonder whether a FS 


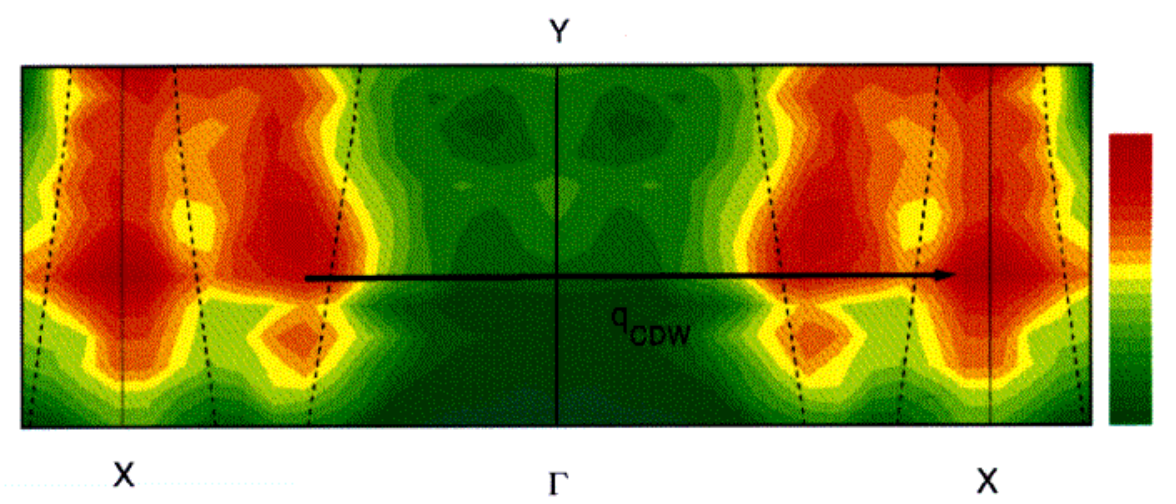

Figure 7. A Fermi energy intensity map for $\mathrm{K}_{0.3} \mathrm{MoO}_{3}$ obtained at $300 \mathrm{~K}$ with $17 \mathrm{eV}$ photons. The overall instrumental resolution was $150 \mathrm{meV}$ FWHM. The intensity increases from green to yellow to red. The measured data are shown in the right-hand half of the map, while the left-hand half was obtained by reflection, a symmetry operation for the crystal. $\Gamma X$ is parallel to the crystallographic $b$-axis, the easy axis. Four almost linear FSs are observed. The dotted lines are the FSs calculated in reference [35] for an $\mathrm{Mo}_{10} \mathrm{O}_{30}$ slab. The measured $\mathrm{CDW}$ wavevector, from reference [40], is shown as an arrow.

can be observed. The answer is 'yes', as shown by the direct FS image for $\mathrm{K}_{0.3} \mathrm{MoO}_{3}$ in figure 7. The detailed procedure for obtaining the image, and other aspects of the ARPES spectra of this material will be reported elsewhere [37]. The actual data set consists of the right-hand half of the image, and the full image has been produced by reflection about the $\Gamma Y$ line. As for the purple bronze image, the details of the intensity distribution along its bright parts arise from the ARPES matrix element. But one can clearly see four lines of the FS, consistent with those expected from band theory, which are shown by the dashed lines. Both of the two experimental pieces of the FS lie closer to the vertical X axis than in the theory. We interpret the experimental feature lying essentially on the vertical $\mathrm{X}$ axis as the unresolved image of the two lines symmetrically disposed on either side of the axis and very close to it. The curvature of the lines shows the departure from one dimensionality, and judging by their centroids, the departures of the experimental features are less than that of the theory. The projection of the experimental CDW wavevector onto the plane of figure 7 is shown by the arrow. The length of the arrow is in excellent agreement with that required for good nesting, i.e. twice the distance from $\Gamma$ to the midpoint of the two pieces of the FS in half of the zone, and is longer than that implied by the theoretical FS. As a final point concerning this figure, it appears visually that the FS image for the blue bronze is more diffuse than that of the purple bronze, which may reflect the lack of weight near $E_{F}$ in the PES spectrum and also in the ARPES EDCs, which we discuss next.

Figure 8 shows a set of EDCs along the quasi-1D $b$-axis of $\mathrm{K}_{0.3} \mathrm{MoO}_{3}$ taken at the SRC at a temperature of $300 \mathrm{~K}$, with a photon energy of $17 \mathrm{eV}$ and energy resolution of $140 \mathrm{meV}$. As expected from the FS image, one sees two peaks which disperse toward $E_{F}$ and are symmetric about the $\mathrm{X}$ point. A previous study [38] observed only the peak nearer to $E_{F}$. Observation of the second peak further from $E_{F}$ is enabled by the very low inelastic backgrounds of our spectra, which we attribute in large measure to taking precautions to prevent the photon-induced sample damage reported by Breuer et al [15]. The dispersion is such as to suggest two crossings of $E_{F}$ near the X point. However, the carefully calibrated position of $E_{F}$ falls on the leading edges of the spectra nearest $E_{F}$ such that their $E_{F^{-}}$ 


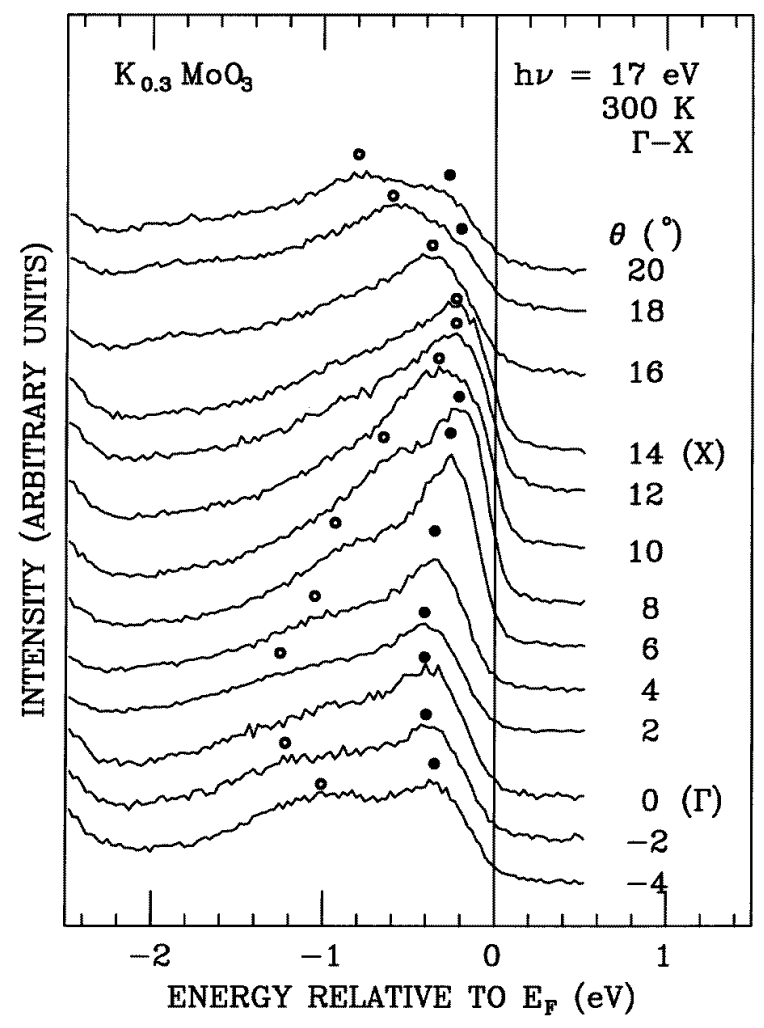

Figure 8. ARPES EDCs for the $\mathrm{K}_{0.3} \mathrm{MoO}_{3}$ conduction band along the easy axis. The measurement was made on a different surface from the one for figure 7, but with other conditions the same.

weights, although non-zero, are anomalously smaller than usually found for $E_{F}$-crossing, e.g. the spectra of figure 2. Thus, in the absence of a direct FS image, and without relying heavily on band theory, the EDCs cannot stand alone as a means of determining the FS. As suggested already, the somewhat diffuse appearance of the FS image probably reflects the $E_{F}$-weight suppression of these unusual lineshapes.

Figure 9 compares the energies of the ARPES peak maxima with the results of the tight-binding band calculation [35]. One sees again the ambiguity of the peak positions as a means of determining $E_{F}$-crossings other than by extrapolation, especially as regards whether there are one or two $E_{F}$-crossings. We have previously [39] called attention to the fact that the low-binding-energy peak is about a factor of two further from $E_{F}$ than the band in the calculation, and that the high-binding-energy peak displays dispersion about five times larger than that of the theoretical band. We have also pointed out [26, 39] that the fast dispersion of the higher-energy peak could be explained in a LL scenario by attributing the peak to the holon feature of the TL spectral function of figure 1. That scenario entailed our finding a quantitative consistency [26] within the TL model, for the case of repulsive spin-independent interactions, between the $\alpha$-value which can be inferred from the onset of the PES spectrum, 0.7-0.9, and the factor-of-five upward renormalization of the holon velocity relative to the underlying single-particle band velocity. In this version of the TL model the spinon velocity is not renormalized, and for this $\alpha$-value the spinon peak is 


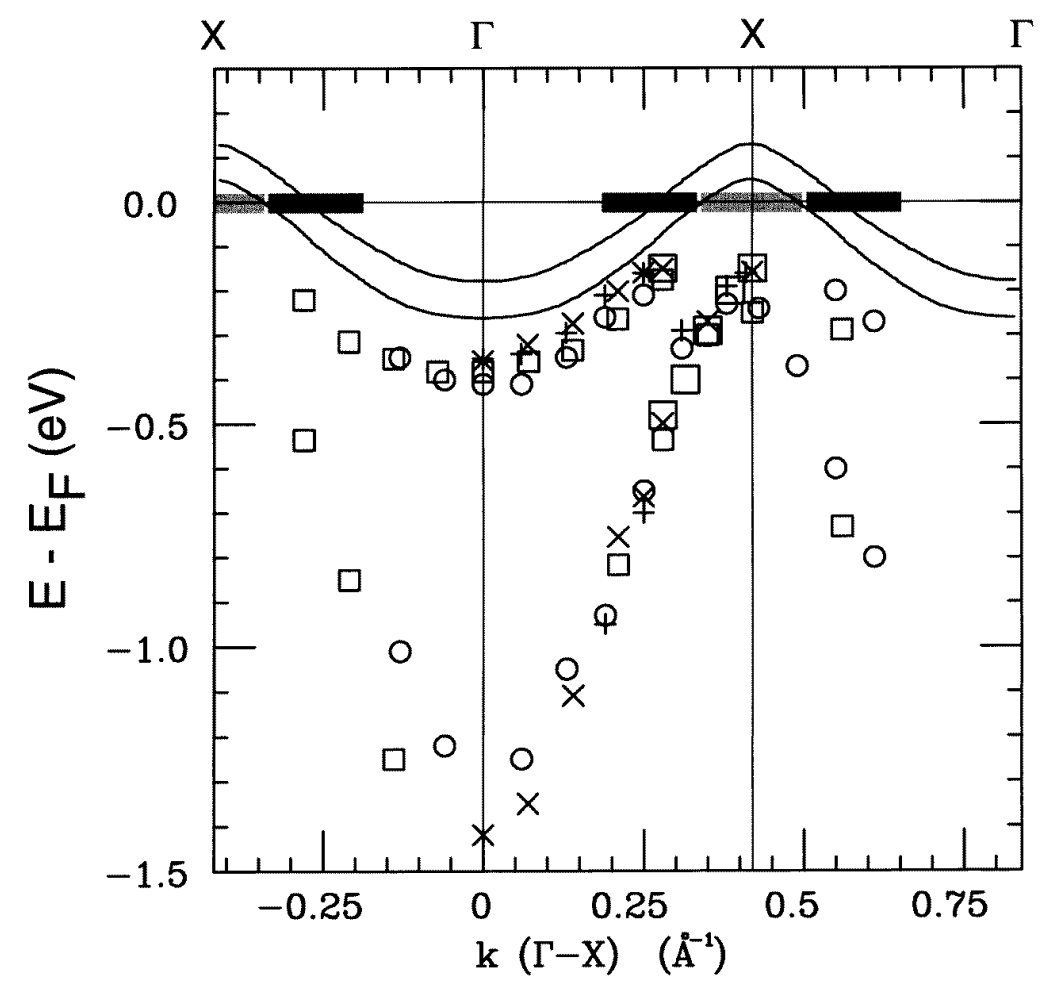

Figure 9. ARPES peak maxima for $\mathrm{K}_{0.3} \mathrm{MoO}_{3}$ compared with the theoretical bands (reference [35]). The bands are calculated for an $\mathrm{Mo}_{10} \mathrm{O}_{32}$ chain and differ slightly from the one that leads to the theoretical FSs shown in figure 7. Circles: SRC, $h v=17 \mathrm{eV}, T=300 \mathrm{~K}$ (figure 8). Crosses: SRC, $h v=20 \mathrm{eV}, T=220 \mathrm{~K}$. Pluses: SRC, $h v=17 \mathrm{eV}, T=220 \mathrm{~K}$. Boxes: SSRL, $h v=20.2 \mathrm{eV}, T=200 \mathrm{~K}$ (small boxes) and $220 \mathrm{~K}$ (big boxes). The CDW transition for this material is at $180 \mathrm{~K}$. The grey bars at the Fermi energy show the location of the $E_{F}$-crossings deduced from figure 7. Approximate FWHMs, parallel to $\Gamma \mathrm{M}$, of the FS 'peaks' in the image are indicated.

suppressed in the TL spectral function. This scenario is admittedly ambiguous as regards the assignment of the low-energy peak, but in the absence of the FS image, and with the unusual lineshapes, this uncertainty could be regarded as acceptable.

Our new results weaken the case for the holon scenario. With the blue bronze FS image in hand, we have a one-to-one relationship between the number of $E_{F}$-crossings, the number of dispersing peaks, and the number of theoretically expected bands. Further, a new band calculation [36] finds dispersion which is still less than that observed, but now only by a factor of two for both bands. The general situation is then almost identical to that of our new results for the purple bronze, where there is also a factor-of-two difference in the occupied bandwidth, but where the $E_{F}$-weight is either not suppressed, or is suppressed on an energy scale too small to resolve. At the moment, in the absence of any further evidence or line of argument, it seems equally possible that one should attribute the bandwidth discrepancy to an error of suitable magnitude in the tight-binding matrix elements, or perhaps to the effects of a $\boldsymbol{k}$-dependent self-energy [41]. Outside the LL scenario, one is then left with CDW fluctuations as the explanation of the nearly zero $E_{F}$ PES spectral weight, but as mentioned above detailed theory does not support this explanation either [14]. 


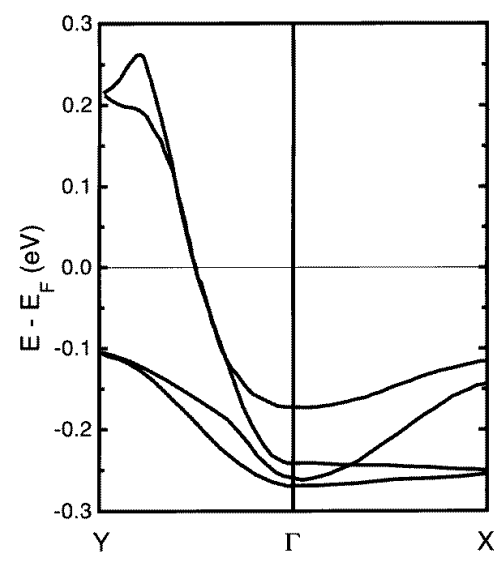

(a)

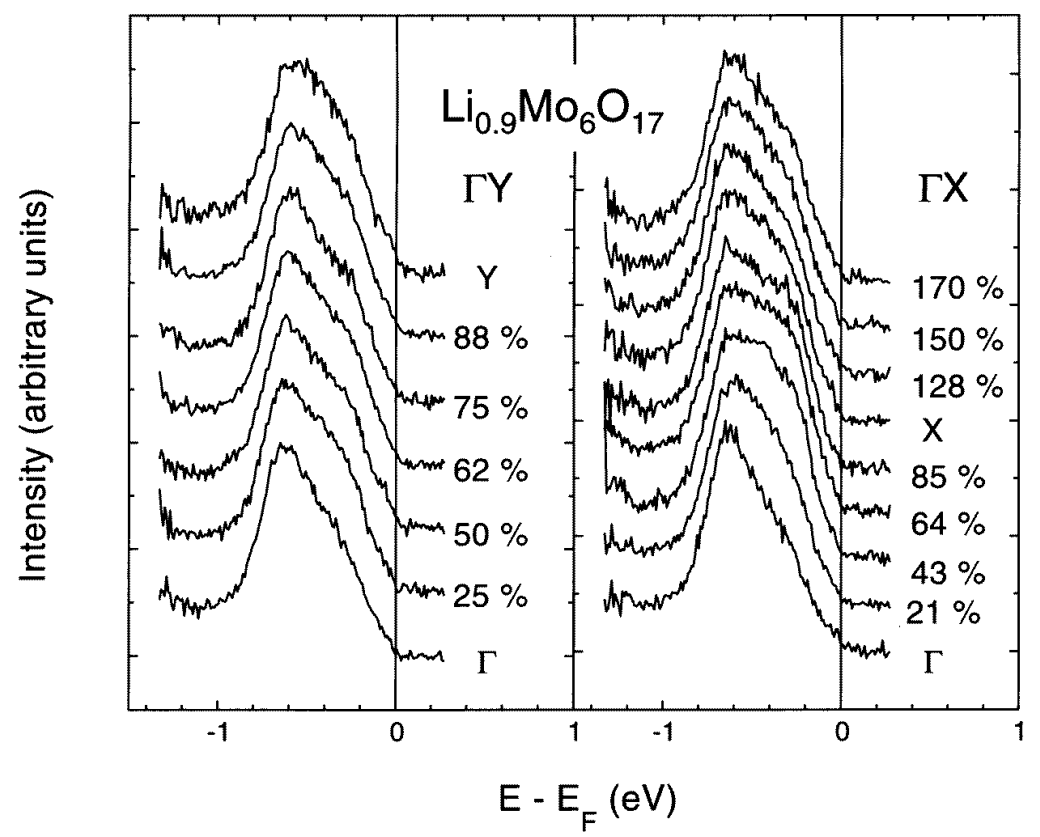

(b)

Figure 10. (a) The tight-binding band calculation for $\mathrm{Li}_{0.9} \mathrm{Mo}_{6} \mathrm{O}_{17}$ from reference [42], showing the conduction bands. (b) ARPES EDCs along $\Gamma Y$ and $\Gamma \mathrm{X}$. The measurement was made at $32 \mathrm{~K}$ with an overall instrumental energy resolution of $43 \mathrm{meV}$ and a photon energy of $20.2 \mathrm{eV}$.

\subsection{Quasi-1D lithium purple bronze}

The so-called Li purple bronze $\mathrm{Li}_{0.9} \mathrm{Mo}_{6} \mathrm{O}_{17}$ is a quasi-1D material which is a superconductor below $T_{c}=2 \mathrm{~K}$. Its transport properties suggest [27] a metal-semiconductor transition at $25 \mathrm{~K}$. Diffraction evidence for either a CDW or a spin-density wave (SDW) does not currently exist, so the exact origin of this transition is not clear. The fact that any $T_{p}$ 
for this material can be no greater than $25 \mathrm{~K}$ is very important because the $300 \mathrm{~K}$ ARPES spectra for this material, as discussed below, show no weight at $E_{F}$. Thus, although the PES spectrum has not yet been measured, it is almost certain that it will not display a Fermi edge at $300 \mathrm{~K}$. The suppression of $E_{F}$-weight at a temperature more than ten times the possible value of $T_{p}$ strongly militates against the CDW fluctuation scenario for this material, and that, in turn, reduces the plausibility of the scenario for the other CDW materials.

As shown in figure 10(a), tight-binding band calculations [42] show an essentially degenerate pair of bands crossing $E_{F}$ along the quasi-1D direction, leading one to expect two lines of the FS. A FS like that predicted has been reported [43], deduced from $300 \mathrm{~K}$ EDCs. The EDCs, reported only for the $\Gamma Y$ direction parallel to the quasi-1D axis, show for all $\boldsymbol{k}$-values a broad background feature which develops a weak shoulder that moves toward $E_{F}$ as one moves away from $\Gamma$. In the vicinity of the $k$-value interpreted as the $E_{F}$-crossing, the weak shoulder loses intensity, leaving again the broad background feature. This background feature can be interpreted as an uncertain mix of a complex lineshape which reflects the total occupied bandwidth, as seems to occur for the $\mathrm{Na}$ and $\mathrm{K}$ purple bronzes, and also of a peak arising from the other bands shown in figure 10(a), which disperse halfway toward $E_{F}$ but do not cross it. In fact, the predicted dispersion of these other bands is roughly consistent with the dispersion of the weak shoulder. Since there is an almost complete absence of spectral weight at $E_{F}$ in all of the spectra, to a degree even greater than that seen in the blue bronze, the main evidence for an $E_{F}$-crossing is the intensity change of the weak shoulder. Intensity changes of this size are known to arise from the variation of the ARPES matrix element and so it is very difficult to be sure that a FS has actually been observed.

Figure 10(b) shows EDCs that we have obtained for $\mathrm{Li}_{0.9} \mathrm{Mo}_{6} \mathrm{O}_{17}$, both along $\Gamma X$ and along $\Gamma Y$. In the hope of observing sharper features and clearer dispersion than that reported previously, the data were taken at a much lower temperature and with considerably better resolution. Unfortunately, although the high-binding-energy onset of the background feature is indeed much sharper than in the data of reference [43], the dispersing shoulder along the parallel direction is no clearer. As with the data of reference [43], there is no weight at $E_{F}$, making an $E_{F}$-crossing for the shoulder very difficult to identify. One can see that the spectra for the perpendicular $\Gamma X$ direction also show a very similar dispersing shoulder. For this direction, where an $E_{F}$-crossing is not expected from the theory, the shoulder must be interpreted as the bands which, for this direction also, disperse halfway to $E_{F}$ but do not cross it. We conclude that, in the absence of a direct FS image, the primary source of confidence that the correct FS has actually been found for this material is not the EDCs that have been obtained, but instead the general success of the tight-binding theory for the Mo bronzes, and perhaps also the simplicity of the predicted FS. We also take cognizance that, as is the case for the blue and purple bronzes, the occupied bandwidth is underestimated in the theory by about a factor of two.

\section{Summary and conclusions}

In summary, the ARPES spectra and direct FS images for the Na purple and blue bronzes agree remarkably well as regards the numbers of bands and the FSs predicted in tightbinding calculations. The same is probably also true for the Li purple bronze, although the actual experimental evidence is weak at the moment. For all three bronzes the experimental occupied bandwidth is at least a factor of two greater than that calculated. The commonality of the bandwidth discrepancy across several materials, and the uncertainty of its exact magnitude, cause us to draw back somewhat from the special holon scenario that we have 
discussed previously for the blue bronze. Perhaps the bandwidth discrepancy signals a systematic error in the tight-binding calculations for these materials.

Nonetheless, the ARPES lineshapes suggest interaction effects of some sort. For the quasi-2D Na purple bronze, where the numbers of dispersing features are as expected, and where there is no suppression of $E_{F}$-weight, the ARPES lineshapes are still not simple peaks. Even in cases where only a single band is present, the lineshape shows substantial weight across the entire occupied bandwidth. With such a large energy scale, if these lineshapes represent the hole spectral function, which we believe to be true for $\mathrm{TiTe}_{2}$, then they imply that in addition to electron-phonon interactions electron-electron interactions are also present.

For the quasi-1D materials one has also $E_{F}$-weight suppression both in ARPES lineshapes and PES spectra. For the blue bronze, the fact that this weight suppression is observed from the same surfaces which yield dispersing ARPES peaks, and a good FS image as well, shows that it is not the result of a damaged surface, as was suggested previously [15], and so should be treated seriously. Indeed $E_{F}$-weight suppression seems to be a clear property of quasi-1D but not quasi-2D materials. Although such weight suppression is a property of the TL spectral function, the measured lineshapes do not show at the qualitative level the other features characteristic of the TL model. At the same time, it is almost certain that the fluctuating-CDW scenario is not applicable to the Li purple bronze, and it is unlikely [14] that it applies to the blue bronze. Thus the primary spectroscopic evidence of NFL behaviour remains the $E_{F}$-weight suppression, and the ARPES data serve to give great credence to this interesting finding, which remains to be adequately explained for these materials.

\section{Acknowledgments}

G-HG and JWA thank M-H Whangbo for useful discussions. We are indebted to Z-X Shen for the use of his end-station and to the members of his group for help and hospitality in the work at SSRL. Work at U-M was supported by the US Department of Energy (DoE) under contract DE-FG02-90ER45416 and by the US National Science Foundation (NSF) grant DMR-91-08015. The work at Universität Saarbrücken was funded by the Deutsche Forschungsgemeinschaft. Work at LANL was supported by the DoE. Work at the Ames Laboratory was supported by the DoE under contract W-7405-ENG-82. The SRC is supported by the NSF and the SSRL is supported by the DoE.

\section{References}

[1] Haldane F D M 1981 J. Phys. C: Solid State Phys. 142585

[2] Luttinger J M 1963 J. Math. Phys. 41154

Mattis D C and Lieb E H 1965 J. Math. Phys. 6304

Luther A and Peschel I 1974 Phys. Rev. B 902911

[3] Meden V and Schönhammer K 1992 Phys. Rev. B 4615753

Schönhammer K and Meden V 1993 Phys. Rev. B 4716205

Voit J 1993 Phys. Rev. B 476740

[4] Smith N V, Thiry P and Petroff Y 1993 Phys. Rev. B 4715476

[5] Anderson P W and Ren Y 1990 High Temperature Superconductivity Proceedings ed K S Dedell, D Coffey, D E Meltzer, D Pines and J R Schrieffer (Redwood City, CA: Addison-Wesley) p 3 Anderson P W 1990 Phys. Rev. Lett. 64 1839; 1990 Phys. Rev. Lett. 65 2306; 1994 Physica B 199+200 8

[6] Varma C M, Littlewood P B, Schmitt-Rink S, Abrahams E and Ruckenstein A E 1989 Phys. Rev. Lett. 63 1996

[7] Dardel B, Malterre D, Grioni M, Weibel P, Baer Y and Lévy F 1991 Phys. Rev. Lett. 673144 
[8] Dardel B, Malterre D, Grioni M, Weibel P, Baer Y, Voit J and Jérôme D 1993 Europhys. Lett. 24687

[9] Bourbonnais C, Creuzet F, Jérôme D, Bechgaard K and Moradpour A 1984 J. Physique Lett. 45 L755 Wzietek P, Creuzet F, Bourbonnais C, Jérôme D, Bechgaard K and Batail P 1993 J. Physique I 3171

[10] Mila F and Zotos X 1993 Europhys. Lett. 24133

[11] Penc K, Mila F and Shiba H 1995 Phys. Rev. Lett. 75894

[12] Lee P A, Rice T M and Anderson P W 1973 Phys. Rev. Lett. 31462 Rice M J and Strässler S 1973 Solid State Commun. 131389

[13] Dardel B, Malterre D, Grioni M, Weibel P, Baer Y, Schlenker C and Pétroff Y 1992 Europhys. Lett. 19525

[14] McKenzie R H 1995 Phys. Rev. B 5216428

[15] Breuer K, Smith K E, Greenblatt M and McCarroll W 1994 J. Vac. Sci. Technol. A 122196

[16] Claessen R, Wilde C, Reinert F, Hüfner S, Gweon G-H, Allen J W, Poirier D M and Olson C G 1996 Phys. Rev. B submitted

[17] Terrasi A, Marsi M, Berger H, Margaritondo G, Kelly R J and Onellion M 1995 Phys. Rev. B 525592

[18] Vincent H, Ghedira M, Marcus J, Mercier J and Schlenker C 1983 J. Solid State Chem. 47113

[19] Schlenker C, Schwenk H, Escribe-Filippini C and Marcus J 1985 Physica B 135511

[20] Wold A, Kunnmann W, Arnott R J and Ferretti A 1964 Inorg. Chem. 3545

[21] Hedin L and Lundqvist S 1969 Solid State Physics vol 23, ed H Ehrenreich, D Turnbull and F Seitz (New York: Academic)

[22] Koike Y, Okamura M, Nakanomyo T and Fukase T 1983 J. Phys. Soc. Japan 52597 Allen P and Chetty N 1994 Phys. Rev. B 5014855

[23] Claessen R, Anderson R O, Allen J W, Olson C G, Janowitz C, Ellis W P, Harm S, Kalning M, Manzke M and Skibowski M 1992 Phys. Rev. Lett. 69808

[24] Claessen R, Anderson R O, Gweon G-H, Ellis W P, Allen J W, Janowitz C, Olson C G, Shen Z X, Eyert V, Skibowski M, Frimelt K, Bucher E and Hüfner S 1996 Phys. Rev. B at press

[25] Straub T, Claessen R, Steiner P and Hüfner S, unpublished

[26] Allen J W, Gweon G-H, Claessen R and Matho K 1995 J. Phys. Chem. Solids 561849

[27] Schlenker C 1989 Low-dimensional Electronic Properties of Molybdenum Bronzes and Oxides (Dordrecht: Kluwer-Academic)

Dumas J and Schlenker C 1993 Int. J. Mod. Phys. B 74045

[28] Onoda M, Matsuda Y and Sato M 1987 J. Solid State Chem. 6967

[29] Whangbo M-H, Canadell E and Schlenker C 1987 J. Am. Chem. Soc. 1096308

[30] Whangbo M-H, Canadell E, Foury P and Pouget J-P 1991 Science 25296

[31] Escribe-Filippini C, Konaté K, Marcus J, Schlenker C, Almairac R, Ayroles R and Roucau C 1984 Phil. Mag. B $\mathbf{5 0} 321$

[32] Schulz H J 1983 J. Phys. C: Solid State Phys. 166769

Fabrizio M and Parola A 1993 Phys. Rev. Lett. 70226

Kopietz P, Meden V and Schönhammer K 1995 Phys. Rev. Lett. 742997

[33] Breuer K, Stagarescu C, Smith K E, Greenblatt M and Ramanujachary K 1996 Phys. Rev. Lett. 763172

[34] Gweon G-H, Clack J A, Zhang Y X, Allen J W, Poirier D M, Benning P J, Olson C G, Marcus J and Schlenker C 1996 to be published

[35] Whangbo M-H and Schneemeyer L F 1986 Inorg. Chem. 252424

[36] Whangbo $\mathrm{M}-\mathrm{H}$, private communication

[37] Gweon G-H, Claessen R, Allen J W, Clack J A, Ellis W P, Reinert F, Poirier D M, Benning P J, Olson C G, Zhang Y X, Matsuura A, Schneemeyer L F and Hüfner S 1996 to be published

[38] Veuillen J Y, Cinti R C and Al Khoury Nemeh E 1987 Europhys. Lett. 3355

[39] Claessen R, Gweon G-H, Reinert F, Allen J W, Ellis W P, Shen Z X, Olson C G, Schneemeyer L F and Lévy F 1995 J. Electron Spectrosc. Relat. Phenom. 76121

[40] Pouget J P, Kagoshima S, Schlenker C and Marcus J 1983 J. Physique 44 L113

[41] Inoue I H, Hase I, Aiura Y, Fujimori A, Haruyama Y, Maruyama T and Nishihara Y 1995 Phys. Rev. Lett. 742539

Inoue I H, Aiura Y, Nishihara Y, Haruyama Y, Nishizaki S, Maeno Y, Fujita T, Bednorz J G and Lichtenberg F 1996 J. Electron Spectrosc. Relat. Phenom. at press

[42] Whangbo M-H and Canadell E 1988 J. Am. Chem. Soc. 110358

[43] Smith K E, Breuer K, Greenblatt M and McCarroll W 1993 Phys. Rev. Lett. 703772 